\title{
Management of Ellis Class II Fracture by Fragment attachment: A Clinical Report
}

\author{
Dr. Reema Malik ${ }^{1}$, Dr. Amit Raj ${ }^{2}$, Dr. Rajul Vivek ${ }^{3}$ \\ ${ }^{I}$ (Department Of Conservative Dentistry And Endodontics, Faculty Of Dental Sciences, IMS, BHU, India) \\ ${ }_{2}^{2}$ (Department Of Prosthodontics, Faculty Of Dental Sciences, IMS, BHU, India) \\ ${ }^{3}$ (Phd Scholar, Faculty Of Dental Sciences, IMS, BHU, India)
}

\begin{abstract}
Coronal fracture of permanent dentition is the most frequent type of dental injury. The immediate agglutination of original fractured tooth is a good alternative option in the scope of emergency treatment for remaining esthetical and functional problems. This article reports the management of Ellis Class II Fractured tooth using adhesive reattachment of the same fragment.
\end{abstract}

Keywords: Crown fracture, Reattachment, Trauma, Ellis Class II Fracture, uncomplicated fracture, Resin composite

\section{Introduction}

The uncomplicated fracture of a crown is absolutely the most common form of traumatic lesion, affecting around $25 \%$ of the population under the age of 18 (1). A majority of these fractures involved the maxillary central incisors, with boys outnumbering girls almost two to one. (1-3).

The treatment of an uncomplicated coronal fracture is a considerable challenge for the dentist because many parameters are implicated in the successful outcomes of the restoration: the necessity to obtain an aesthetic result that nears itself to the natural form and dimension, the opacity and translucency, the fluorescence and opalescence of the original tooth.

Several techniques have been advocated for the restoration of fractured teeth, such as resin, ceramic or steel crowns, orthodontic bands, and resin composite restorations with and without pins (4). However, these types of treatments did not always guarantee an adequate long-lasting aesthetic result and required substantial sacrifice of the dental structure during the preparation.

Recent developments in restorative materials, placement techniques, preparation design, and an adhesive protocol facilitates restoration of fractured maxillary incisors by fragment reattachment. Tennery was the first to report the re-attachment of a fractured fragment using acid-etch technique (5). Subsequently, Starkey and Simonsen have reported similar cases $(6,7)$.

The reattachment of the fractured crown fragments using the bonding fragment technique offers several advantage including the re-establishment of function, esthetics, shape, shine and surface texture in a short time, there by preserving the original contour and alignment of dental tissues.

This paper reports a case of Ellis Class II Fractured tooth managed using reattachment of the same fragment.

\section{Case Report}

A 7-year-old patient reported to the department of conservative dentistry and endodontics following fracture of the crown in the left maxillary central incisor. The fractured tooth segments was recovered at the site of the injury and placed in water. The trauma had occurred due to a fall about 2 hours ago.

The intraoral and radiographic examination showed that the injury caused a non complicated crown fracture in Maxillary left central incisor and jagged enamel margins in right maxillary central incisor (Fig 1a, 1b). Clinical examination evidenced a fracture involving the enamel/dentin aspect with no symptoms. The crown fragment analysis showed a perfect margin adaptation of the fragment to the tooth remnant.

The position and pattern of the fracture, the occlusion (maxillo-mandibular relationship) and a tooth remnant with an intact substrate suggested that pulp protection followed by reattachment of the fragment to its original position using adhesives procedures was a reliable option for the case. The patient was systemically healthy, presented an overall plaque index and gingival index compatible with good periodontal health, and the operative area was free of visible plaque.

The fractured fragment was stored in physiological saline for the time being (Fig 2a, 2b). After the placement of rubber dam, the glass ionomer cement base was given covering the exposed dentine, excess cement was carefully trimmed, so as not to touch the margins of the fracture line, in an attempt to obtain an adequate seating of the fragment to the tooth remnant. The adaptation of the fragment was checked. Phosphoric acid gel 37.5\% (Scotch BondTM, 3M ESPE, St. Paul, USA) was applied to the enamel of the fragment and the teeth for 20 seconds, limited to $2 \mathrm{~mm}$ beyond the fracture margin. Air-water spray was used to remove the acid 
and the surface was air-dried. An adhesive system (Adper Single Bond PlusTM, 3M ESPE) was applied to the tooth fragment. A small increment of resin composite was applied to the tooth fragment which was then reattached to its proper position. Visible light polymerization was done for 60 seconds to the facial and palatal surfaces of the tooth, while the fragment was kept in position under pressure. The tooth was polished with polishing discs (Shofu) and the rubber dam was removed. The Jagged enamel edge of right maxillary central incisor was smoothened using polishing discs (Fig 3a, 3b, 3c).

Clinical and radiographic examination was carried out after 3 month \& 6 months (Fig 4a, 4b). Teeth responded positively to the pulp vitality tests and the radiographs showed no periapical changes.

\section{Discussion}

The fracture of a tooth may be a most traumatic incident for a young patient, but it has been found that there is a positive emotional and social response from the patient to the preservation of natural tooth structure (8).

The dentist plays an important role in the management of injured cases and so he has to take into consideration every possibility of saving a tooth that has received trauma. The remarkable advancement of adhesive systems and resin

composites have made reattachment of tooth fragments a procedure that is no longer a provisional restoration, but rather a restorative treatment offering a favourable prognosis. However, this technique can be used only when the intact tooth fragment is available (9).

In the present case, the location and aspect of the fracture (non-complicated crown fracture) and the presence of a balanced occlusion may have favoured the clinical success. Limitations in the bonding fragment technique are attributed to detachment of the remaining dental fragment; the fragment does not recover its original colour or bonding of the remaining fragment at the incorrect position. Fortunately, during the entire follow-up period, none of these adverse conditions occurred in the patient.

The inflammatory process in cases of coronal fractures with minor dentin involvement is transitory, since the vascular supply of the pulp remains intact and bacterial invasion can be prevented. The vascular supply depends fundamentally on the trauma occurred, however, bacterial invasion can be prevented by immediate dentin sealing. [9] The use of natural tooth substance clearly eliminated problems of differential wear of restorative material, unmatched shades and difficulty of contour and texture reproduction associated with other restorative techniques.

\section{Conclusion}

Tooth fragment reattachment procedure offers an ultraconservative, safe, fast and esthetically pleasing result when the fractured fragment is available. Reattachment of the dental fragment as a restorative procedure has become possible with the improvement of adhesive techniques and restorative materials

\section{References}

[1]. Andreasen JO, Ravn J J. Epidemiology of traumatic dental injuries to primary and permanent teeth in a Danish population sample. Int J Oral Surg 1972;1(5):235-239

[2]. Zerman N, Cavalleri G. Traumatic injuries to permanent incisors. Endodont Dent Traumatol 1993;9(2):61-64.

[3]. Martens LC, Beyls HM, de Craene LG, D'Hauwers RF. Reattachment of the original fragment after vertical crown fracture of a permanent central incisor. J Pedod 1988;13(1):53-62.

[4]. Simonsen R., Thompson V.P., Barrark G.: Etched cast restorations: clinical and laboratory technique. Chicago, Quintessence Publishing Co. 1985: 150 - 151.

[5]. Tennery N.T.: The fractured tooth reunited using the acid etch bonding technique. Tex Dent J 1988; 96: $16-17$

[6]. Starkey P.E.: Reattachment of a fractured fragment to a tooth. J Ind Dent Assoc 1979; $58: 37$ - 38.

[7]. Simonsen R.J.: Restoration of a fractured central incisor using original teeth. J Am Dent Assoc 1982; 105: 646 - 648

[8]. Baratieri L.N., Monteiro S.: Tooth fracture reattachment: Case reports. Quint Int 1990; 21: 261 - 270.

[9]. Simonsen R.J.: Traumatic fracture restorations: An alternative use of the acid etch technique. Quint Int. 1979; 10(2): 15 - 22.

[10]. Robertson A, Andreasen FM, Bergenholtz G, Andreasen JO, Munksgaard C. Pulp reactions to restoration of experimentally induced crown fractures. J Dent 1998;26:409-16.

\section{Vi. Images}

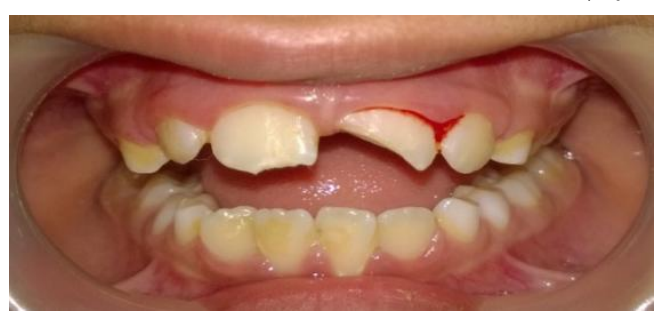

Fig 1a. Pre-operative Frontal view

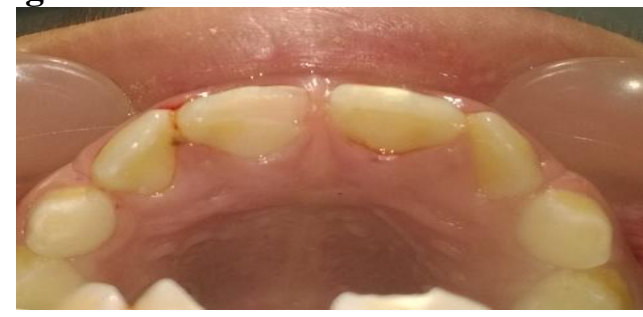

Fig 1b. Pre-operative Palatal View 


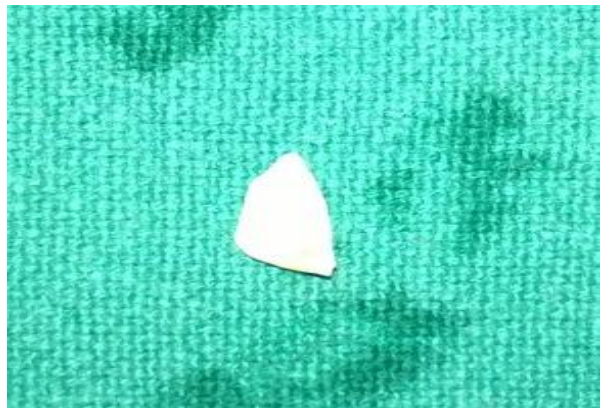

Fig 2a. Fractured segment

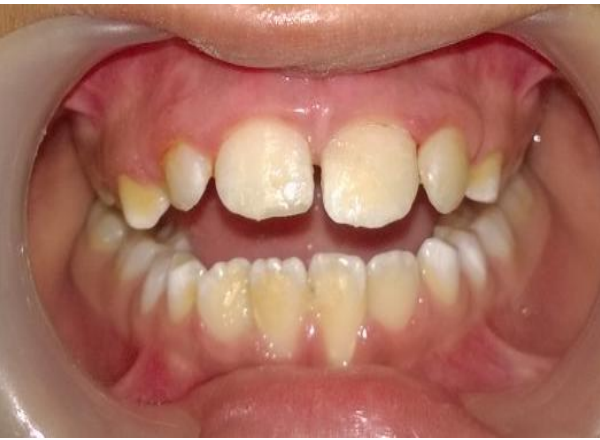

Fig 3a. Post operative Clinical View after Fractured segment reattachment

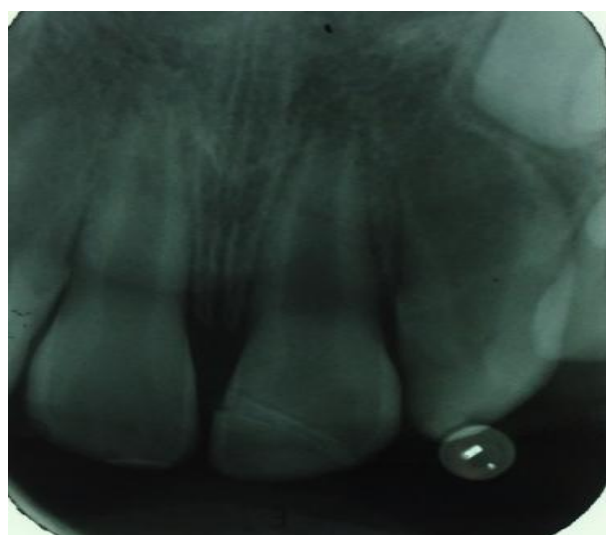

Fig 3c. Post-operative IOPAR

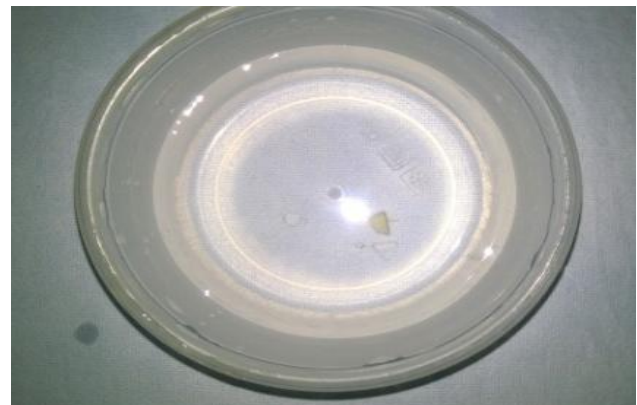

Fig 2b. Fractured segment stored in Normal Saline

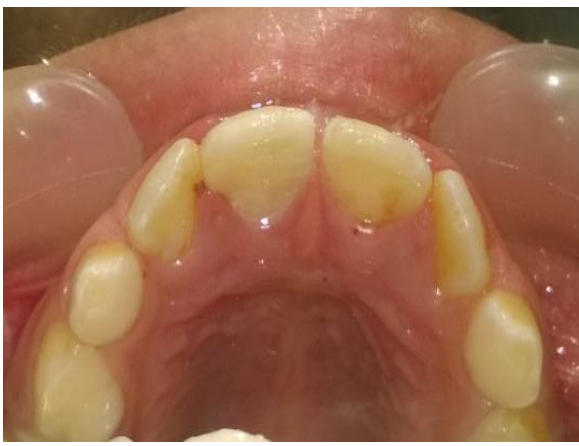

Fig 3b. Post operative Palatal Viaw

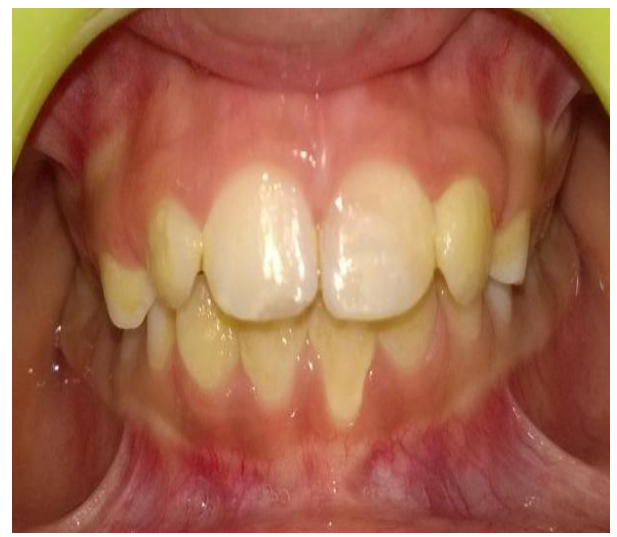

Fig 4a. 3 months follow up

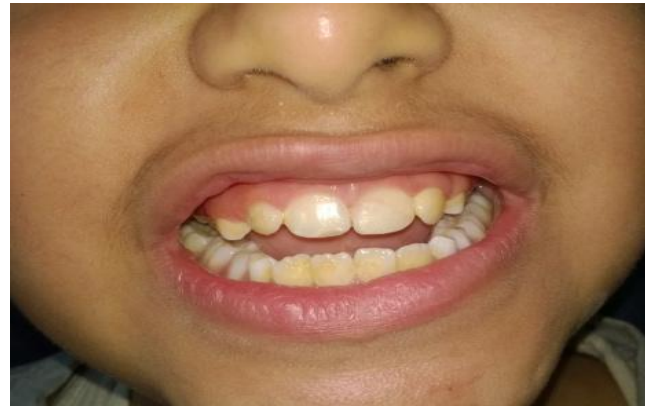

Fig 4b. 6 months smile View 